\title{
THEORETICAL GROWTH AND EQUILIBRIUM FORMS OF ADP $\left(\mathrm{NH}_{4} \mathrm{H}_{2} \mathrm{PO}_{4}\right)$
}

\author{
M. AGUILÓ \\ Department of Crystallography, Faculty of Chemistry in Tarragona, University of Barcelona, Plaça Imperial Tàrraco 1 , \\ 43005 Tarragona, Spain
}

and

\section{C.F. WOENSDREGT}

Department of Crystallography, Institute of Earth Sciences, State University of Utrecht, P.O. Box 80.021, 3508 TA Utrecht, The Netherlands

Received 24 June 1986; manuscript received in final form 24 March 1987

The Hartman-Perdok theory enables one to establish the $F$ forms of ammonium dihydrogen phosphate (ADP) as $\{100\}$ and $\{011\}$. Although the $\{0 k l\}$ forms are theoretically expected to be $\mathrm{S}$ faces, they nevertheless exhibit $\mathrm{K}$ face characteristics for $k<l$. The forms $\{0 \mathrm{kl}\}$, with $k>l$, which are typical of the so-called tapering of the ADP crystals, are S faces. The attachment energy of a particular face, which is assumed to be directly proportional to its growth rate, can be computed in an electrostatic point charge model. As the ionic charge distribution in the crystallizing units is not known exactly, the calculations have been made in 36 models, with $\mathrm{P}-\mathrm{O}$ and $\mathrm{N}-\mathrm{H}$ bond varying from purely ionic to completely covalent. The theoretical growth forms comprise always the $\{011\}$ and sometimes $\{100\}$. The presence of the latter is a function of the ionic charge of the oxygen, $q_{\mathrm{O}}$, and that of the hydrogen $\mathrm{H}_{0}$ belonging to the $\left[\mathrm{H}_{2} \mathrm{PO}_{4}\right]^{-}$group, $q_{\mathrm{H}_{0}}$. The form $\{100\}$ is present, if $-0.9<q_{\mathrm{O}}<0$, when $q_{\mathrm{H}_{0}}$ varies accordingly between 0 and 0.8 . When halving of the elementary growth layer $d_{011}$ is applicable, the theoretical growth forms of all the models show $\{100\}$ and $\{011\}$ resulting in a very pronounced prismatic habit. The presence of impurities can explain the occurrence of $\{031\}$ on the growth forms of all the models, which therefore resemble the so called tapered ADP crystals. The equilibrium forms show always $\{100\}$ and $\{011\}$. The prismatic habit is more pronounced for models with a low $q_{\mathrm{O}}$ and $q_{\mathrm{H}_{0^{*}}}$. The hydrogens $\mathrm{H}_{0}$ are situated just on the boundaries of the elementary growth layers. The effect of ordering of these $\mathrm{H}_{0}$ ions on the theoretical growth and equilibrium forms is not substantial.

\section{Introduction}

\subsection{Previous work}

The crystal morphology of the important ferro-electric and electro-optic component ADP $\left(\mathrm{NH}_{4} \mathrm{H}_{2} \mathrm{PO}_{4}\right)$ has recently been deduced from its crystal structure by Aguiló and Woensdregt [1]. They establish the order of morphological importance of the ADP crystal faces by identifying the PBCs consisting of $\mathrm{N}-\mathrm{H}_{\mathrm{N}} \ldots \mathrm{O}$ strong bonds between the ionic $\left[\mathrm{NH}_{4}\right]^{+}$and $\left[\mathrm{H}_{2} \mathrm{PO}_{4}\right]^{-}$crystallizing units. These PBCs are parallel to $\langle 100\rangle$, [001], $\left\langle\frac{1}{2} \frac{1}{2} \frac{1}{2}\right\rangle$ and $\left\langle\frac{1}{2} \frac{1}{2} \frac{3}{2}\right\rangle$. F faces which are parallel to at least two PBCs and grow slowly according to a layer mechanism are $\{011\}$ and $\{100\}$. S forms parallel to only one $\mathrm{PBC}$ are $\{112\}$ and $\{110\}$. The $\{0 k l\}$ faces with $k>l$, which are characteristic of the so-called tapering of ADP crystals, are S faces. According to the Hartman-Perdok theory [2], only $F$ faces should be present on crystals as faces. The crystals of ADP show normally a prismatic habit with the prism $\{100\}$ and the bipyramid \{011\}. In addition, the crystal forms $\{0 k l\}$ with $k>l$, producing the tapering, occur sometimes under special conditions [3]. In the present paper not only the morphological importance of the $F$ faces will be quantified, but also quantitative information about the $S$ faces $(0 \mathrm{kl})$ with $k>l,\{110\}$ and $\{112\}$ will be provided. 


\subsection{Attachment and other related energies}

The PBC analysis defines the slowly growing $F$ faces in a qualitative way. Depending on its growth rate, an $\mathrm{F}$ face may or may not actually appear on a growing crystal. It is therefore necessary to establish the growth rates of all $\mathrm{F}$ faces. Hartman [2] defined the attachment energy $E_{\mathrm{a}}$ as the energy released per molecule, when a new slice of thickness $d_{h k l}$ crystallizes on an already existing crystal face $(h k l)$. Hartman and Bennema [4] indicated that for an $\mathrm{F}$ face the attachment energy is directly proportional to the growth rate. Once the attachment energies of all the $F$ faces are known, a growth form can be constructed following the principles of the so-called Wulff plot (see for more information Woensdregt [5]). The lower the attachment energy, the higher probability that an $F$ face will be present. The geometrical relation to other faces is, however, also an important factor which determines the presence of an $F$ form on the growth form [5]. The growth, and later also the equilibrium, forms have been plotted, all having the same volume using the APL program Crystaldraw [6].

The attachment energy $E_{\mathrm{a}}$ is the sum of all the partial energies $E_{m}$ over all $m$ slices into which a crystal has been divided parallel to the crystal face $(h k l)$ :

$E_{\mathrm{a}}=\sum_{m=1}^{\infty} E_{m}$.

The slice energy $E_{\mathrm{s}}$ is the energy released per molecule when a new slice $d_{h k l}$ is formed from the vapour neglecting edge energies. As the lattice energy is the energy released per molecule when the crystal crystallizes from the vapour, the following relation holds:

$E_{\mathrm{cr}}=E_{\mathrm{a}}+E_{\mathrm{s}}$.

It is also possible to define the chain energy of a $\mathrm{PBC}$ as the energy released per molecule, when it is formed from the vapour. Although the chain energy varies according to the PBC configuration, it might be used as an indication of the relative importance of the PBC.

Calculations of attachment energies have been carried out in an electrostatic point charge model, which has been described earlier by Hartman [7] for the cubic sphalerite crystal structure. This model modified and adapted for all the crystal systems has been used in our calculations of the attachment, chain and slice energies [8].

The atomic coordinates and unit cell data are the same as used in the previous qualitative study [1]. The energies are given in $\mathrm{kJ} \mathrm{mol}^{-1}$ on the basis of $1 \mathrm{e}^{2} \AA^{-1}=1389.3 \mathrm{~kJ} \mathrm{~mol}^{-1}$.

\subsection{Models}

In order to calculate the attachment energies in an electrostatic point charge model, the effective charge of each individual atom has to be known. In a saturated solution having a $\mathrm{pH}$ of about 4.0 , ADP is completely dissociated into $\left[\mathrm{NH}_{4}\right]^{+}$and $\left[\mathrm{H}_{2} \mathrm{PO}_{4}\right]^{-}$ions [9]. The bonds within the $\left[\mathrm{NH}_{4}\right]^{+}$ and $\left[\mathrm{H}_{2} \mathrm{PO}_{4}\right]^{-}$ions are neither completely ionic nor covalent. On the basis of the SCF ab initio theory Pullman et al. [10] calculated the following charge distribution for the $\left[\mathrm{H}_{2} \mathrm{PO}_{4}\right]^{--}: \mathrm{P}=+0.852$, $\mathrm{O}=-0.613$ and $\mathrm{H}=+0.300$. According to the formula given by Scorzyck [11], the charge distribution of the $\left[\mathrm{H}_{2} \mathrm{PO}_{4}\right]^{-}$ion should be $\mathrm{P}=+3.52$, $\mathrm{O}=-1.445$ and $\mathrm{H}=+0.63$.

The attachment energies have been calculated in 36 different models, not only because the exact charge distribution is apparently still subject to discussions, but also in order to see whether that distribution would have an effect on the theoretical crystal morphology.

In table 1 the different charge distributions with their corresponding labels are summarized. Models with $\mathrm{O}^{2-}, \mathrm{O}^{-}$and $\mathrm{O}^{0}$ are labelled respectively $\mathrm{A}^{* *}, \mathrm{~B}^{* *}$ and $\mathrm{C}^{* *}$. The models with different effective charges for the hydrogen $\mathrm{H}_{0}$ of the $\left[\mathrm{H}_{2} \mathrm{PO}_{4}\right]^{-}$ion are labelled ${ }^{*} \mathrm{a} *,{ }^{*} \mathrm{~b}^{*}$ and ${ }^{*} \mathrm{c} *$ when $q_{\mathrm{H}_{0}}$ equal respectively $+1,+0.5$ and +0.25 . Finally the hydrogen $\mathrm{H}_{\mathrm{N}}$ of the $\left[\mathrm{NH}_{4}\right]^{+}$ion of the models labelled $* * 1,{ }^{*} 2,{ }^{* *} 3$ and ${ }^{* *} 4$ assumes respectively the effective charges $+1,+0.5,+0.25$ and 0 . Within the $\left[\mathrm{H}_{2} \mathrm{PO}_{4}\right]^{-}$ion the covalent character varies from 0 to $100 \%$ for the $\mathrm{P}-\mathrm{O}$ bonds in the models $\mathrm{A}^{* *} \rightarrow \mathrm{C}^{* *}$, as well as for the $\mathrm{N}-\mathrm{H}_{\mathrm{N}}$ bonds of the $\left[\mathrm{NH}_{4}\right]$ ions in the models ${ }^{* *} 1 \rightarrow * * 4$. The hydrogen bonds $\left(\mathrm{N}-\mathrm{H}_{\mathrm{N}} \ldots \mathrm{O}\right.$ and 
Table 1

Valences used for the calculation of energies in the different point charge models of ADP

\begin{tabular}{lrllllll}
\hline Model & $q_{0}$ & Model & $q_{\mathrm{H}_{0}}$ & $q_{\mathrm{PO}}$ & Model & $q_{\mathrm{H}_{\mathrm{N}}}$ & $q_{\mathrm{N}}$ \\
\hline $\mathrm{A} \cdot \cdots$ & -2 & ${ }^{*} \mathrm{a}^{*}$ & 1 & -3 & ${ }^{*} 1$ & 1 & -3 \\
$\mathrm{~B} \cdots$ & -1 & ${ }^{*} \mathrm{~b}^{*}$ & 0.5 & -2 & $* * 2$ & 0.5 & -1 \\
$\mathrm{C} \cdot$ & 0 & ${ }^{*} \mathrm{c}^{*}$ & 0.25 & -1.5 & $* * 3$ & 0.25 & 0 \\
& & & & & $* * 4$ & 0 & 1 \\
\hline
\end{tabular}

$\left.\mathrm{O}-\mathrm{H}_{0} \ldots \mathrm{O}\right)$ are progressively weaker in the model series $* \mathrm{a}^{*} \rightarrow{ }^{*} \mathrm{c}^{*}$ and ${ }^{* *} 1 \rightarrow * * 4$.

\subsubsection{Model Aal}

First of all, calculations have been carried out in model Aa1, which has the normal charges $q_{\mathrm{O}}=$ -2 , and $q_{\mathrm{H}_{0}}=1$, i.e. $\left[\mathrm{N}^{3-} \mathbf{H}_{4}^{+}\right]^{+}\left[\mathrm{H}_{2}^{+} \mathrm{P}^{5+} \mathrm{O}_{4}^{2-}\right]^{-}$, in order to obtain an idea about the absolute values of the attachment energies related with the different crystal faces. These attachment energies are listed in table 2. The F faces have the lowest attachment energies, while the $\mathrm{S}$ faces, such as $\{112\}$ and $\{110\}$, and the $K$ faces $\{013\}$ have much higher attachment energy values. The $S$ face $\{031\}$ has a relatively low attachment energy, certainly if compared with that of the F prism $\{100\}$. Because $\mathbf{S}$ and $\mathbf{K}$ forms other than $\{031\}$ are not likely to have attachment energies comparable with those of $\mathrm{F}$ faces, the decision has been taken to compute only the attachment energies of $\{011\}$, $\{100\}$ and $\{031\}$ in all of the 36 models.

Table 2

Surface energies of ADP crystal faces (model Aa1)

\begin{tabular}{lllll}
\hline$(h k l) /[U V W]$ & Type & $\begin{array}{l}d_{h k l} \\
(\mathrm{~nm})\end{array}$ & $\begin{array}{l}E_{\mathrm{a}} \\
\left(\mathrm{kJ} \mathrm{mol}^{-1}\right)\end{array}$ & $\begin{array}{l}\gamma \\
\left(\mathrm{mJ} \mathrm{m}^{-2}\right)\end{array}$ \\
\hline$(011) /[100]$ & $\mathrm{F}$ & 0.5321 & -450 & -1869 \\
$(01 \overline{1}) /\left[\frac{1}{2} \frac{1}{2} \frac{1}{2}\right]$ & $\mathrm{F}$ & 0.5321 & -450 & -1869 \\
$(011) /[100]_{\mathrm{B}}$ & $\mathrm{F}$ & 0.5321 & -1185 & -4904 \\
$(020) /[100]$ & $\mathrm{F}$ & 0.3750 & -825 & -2410 \\
$(020) /[001]$ & $\mathrm{F}$ & 0.3750 & -825 & -2410 \\
$(031) /[100]_{\mathrm{A}}$ & $\mathrm{S}$ & 0.2373 & -869 & -2409 \\
$(\overline{1} \overline{1} 2) /\left[\frac{1}{2} \frac{1}{2} \frac{1}{2}\right]$ & $\mathrm{S}$ & 0.3075 & -1055 & -2377 \\
$(013) /[100]_{\mathrm{C}}$ & $\mathrm{K}$ & 0.2386 & -1312 & -2605 \\
$(2 \overline{2} 0) /[001]$ & $\mathrm{S}$ & 0.2652 & -1709 & -3546 \\
\hline
\end{tabular}

It has already been shown in the previous qualitative analysis that the slice $d_{011}$ could be defined in two different ways, see also fig. $2 \mathrm{~d}$ in ref. [1]. In the first configuration, called $\mathrm{A}$, the slice boundary is occupied by $\left[\mathrm{NH}_{4}\right]^{+}$ions, and in the second one, marked $\mathrm{B}$, by $\left[\mathrm{H}_{2} \mathrm{PO}_{4}\right]^{-}$ions. The attachment energy of slice $\mathrm{B}$ is about 2.5 times higher than that of the A type slice. Hence the $F$ slice configuration bounded by $\left[\mathrm{NH}_{4}\right]^{+}$ions (type A) is energetically the more favourable one. The same principle applies to the $\mathrm{S}$ slice configuration of $d_{031}$. For that reason the (031) and (011) attachment energies have been calculated in all the remaining models based on the $[100]_{\mathrm{A}} \mathrm{PBC}$, which is energetically the most convenient configuration.

\subsubsection{Effect of the covalent character of the $N-H_{N}$ bond}

The effect of the decrease in the strength of the $\mathrm{N}-\mathrm{H}_{\mathrm{N}} \ldots \mathrm{O}$ bond is not very strong. The ratio $E_{\mathrm{a}}^{010} / E_{\mathrm{a}}^{011}$ which is decisive for the appearance of $\{100\}$ in the presence of $\{011\}$ varies by about $2 \%$, with the exception of two series of models $\left(\mathrm{Cb}^{*}\right.$ and $\left.\mathrm{Cc}^{*}\right)$ in which the $\{100\}$ is already present. In the latter case the variation is of the order of $2 \%$ to $7 \%$. In the present paper only the results of the $* * 2$ models, which correspond to the charge distribution $\left[\mathrm{N}^{-} \mathrm{H}_{4}^{+0.5}\right]^{+}\left[\mathrm{H}_{2} \mathrm{PO}_{4}\right]^{-}$, are listed.

\subsection{PBC Chain energies}

The chain energies of the PBCs are listed in table 3. The values obtained for the different configurations of the [100] PBC demonstrate that the configuration of the complete $\mathrm{PBC}$ is more important than its orientation or its period.

Table 3

ADP periodic bond chain energies (model Aa1)

\begin{tabular}{llll}
\hline$[U V W]$ & $(h k l)$ & Period $(\mathrm{nm})$ & $E_{\mathrm{ch}}\left(\mathrm{kJ} \mathrm{mol}^{-1}\right)$ \\
\hline$\left[\frac{1}{2} \frac{1}{2} \frac{1}{2}\right]$ & $(01 \overline{1})$ & 0.65093 & -41621 \\
{$[100]_{\mathrm{A}}$} & $(010)$ & 0.74997 & -41787 \\
{$[100]_{\mathrm{B}}$} & $(011)$ & 0.74997 & -41031 \\
{$[100]_{\mathrm{C}}$} & $(013)$ & 0.74997 & -41336 \\
{$[001]$} & $(010)$ & 0.75494 & -41399 \\
{$\left[\frac{1}{2} \frac{1}{2} \frac{3}{2}\right]$} & $(301)$ & 1.25043 & -41129 \\
\hline
\end{tabular}


The $\left[\frac{1}{2} \frac{1}{2} \frac{1}{2}\right],[001]$ and $\left[\frac{1}{2} \frac{1}{2} \frac{3}{2}\right]$ PBCs have chain energy values between the minimum and maximum values of those obtained from the different [100] PBC configurations.

\section{Growth forms}

\subsection{The $H_{0}$ boundary ions}

In calculating the attachment energy of the slice $d_{020}$, which is the elementary growth layer of (010), the boundary ions can be selected in two possible ways.

In order to obtain the stoichiometric composition, either four half ions $\mathrm{H}_{0}$ are selected or only half of that number as complete ions (fig. 1a). Selecting all four ions as half ions means that the occupancy of each site is 0.5 . This is a completely disordered boundary condition, because the statistical probability to locate an ion is the same for each ion, i.e. $50 \%$. The other configuration with only half the sites occupied by full ions is, however, a completely ordered situation. At one site the probability is $0 \%$ (vacancy), while at the other it is $100 \%$ (fig $1 \mathrm{~b}$ ). The slice of $(010)$ schematically represented in fig. $1 \mathrm{~b}$ can moreover have four

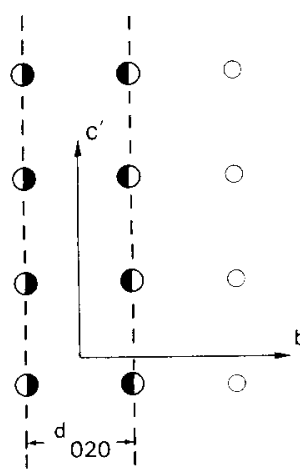

(a)

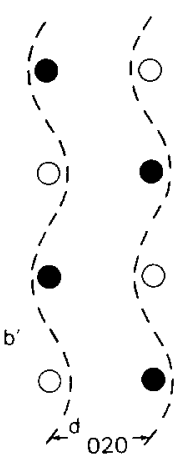

(b)
Fig. 1. In the [100] projection (see also fig. $2 d$ of ref. [1]) the slice $d_{020}$ can have two different surface configurations. This is due to the special position of te $\mathrm{H}_{0}$ hydrogens, which are just on the boundaries of the slices: (a) disordered, hydrogen occupancy of all sites equals 50\% (half open circles); (b) ordered, hydrogen occupancy of half of the sites is equal to $100 \%$ (solid circles), and the other half to $0 \%$ (open circles as vacancies) different ordered configurations, each of which has a different pattern of vacancies.

Fig. 2 summarizes schematically the different (010) configurations which are illustrated by the projection of the $\mathrm{H}_{0}$ hydrogen boundary ions on the (010) surface. The effect on the attachment energy can be calculated by the method of 't Hart [12]. The ordering of the boundary ions reduces the attachment energies. The highest correction must be made for the ordered (i) configuration given in fig. $2 b$. Therefore only the values of this ordered model are listed in table 4 . The energies of the other ordered models lie between those of the disordered and ordered (i) ones. The ordering of the $\mathrm{H}_{0}$ ions is not only to be taken into account for the $\{010\}$, but also for the $\{011\}$ and $\{031\}$ slice configurations. Although the ordering (i) is for all these forms the energetically most favoura-

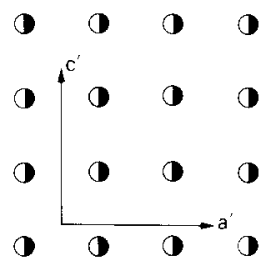

(a)

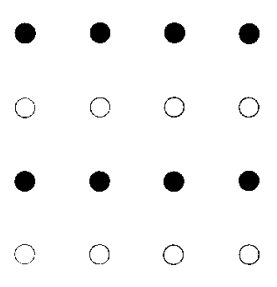

(b)

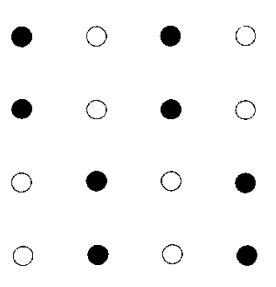

(d)

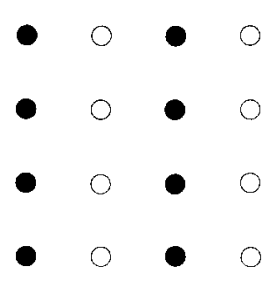

(c)

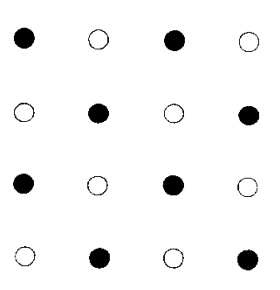

(e)
Fig. 2. Projection of the hydrogen atoms on (010) of ADP showing the different ordering patterns: (a) disordered; (b) ordered (i); (c) ordered (ii); (d) ordered (iii); (e) ordered (iv). 
ble one, the order of increasing attachment energy is not the same, e.g. $\{100\}$ (i), (iv), (iii), (ii); $\{011\}$ : (i), (ii), (iii), (iv).

\subsection{Disordered configurations}

For all the $* * 2$ models the values of attachment energies of the crystal faces (010), (011) and (031) are given in table 4a. Woensdregt [5] introduced the hypermorphological index for a crystal face $(h k l)$. This index (HI) is equal to the minimal decrease in percentage of the attachment energy necessary for that particular face to be present on the growth and equilibrium forms as a crystal face. Hence the HI provides direct information about the relative attachment or surface energies and the geometrical factor, both of which, control the presence of a crystal face. In table 4 an asterisk $\left({ }^{*}\right)$ means that the listed face is present, whereas a negative value of $\mathrm{HI}$ indicates how large a reduction in percentage would permit that face to be present on the theoretical growth or equilibrium form. As the crystal form $\{011\}$ is always present, the HI of that face is not given. One has to keep in mind that the $\mathrm{HI}$ is given for the actual theoretical growth or equilibrium form. Once a new crystal form is included, all the original values of $\mathrm{HI}$ have to be recalculated. Using the

Table 4

Surface energies of ADP, ordered and disordered (**2 models)

(a) Attachment energy (in $\mathrm{kJ} \mathrm{mol}^{-1}$ )

\begin{tabular}{|c|c|c|c|c|c|c|c|c|c|c|c|}
\hline \multirow[t]{4}{*}{ Model } & \multicolumn{11}{|c|}{ Growth form } \\
\hline & \multicolumn{6}{|c|}{ Disordered } & \multicolumn{5}{|c|}{ Ordered (i) } \\
\hline & \multicolumn{2}{|l|}{$(011)$} & \multicolumn{2}{|l|}{$(010)$} & \multicolumn{2}{|l|}{$(031)$} & \multirow{2}{*}{$\begin{array}{l}011) \\
E_{\text {a }}\end{array}$} & \multicolumn{2}{|l|}{$(010)$} & \multicolumn{2}{|l|}{$(031)$} \\
\hline & $E_{\mathrm{a}}$ & $E_{\mathrm{a}}^{* \text { a) }}$ & $\overline{E_{\mathrm{a}}}$ & $\mathrm{HI}$ & $E_{\text {a }}$ & $\mathrm{HI}$ & & $\overline{E_{\mathrm{a}}}$ & HI & $\overline{E_{\mathrm{a}}}$ & $\mathrm{HI}$ \\
\hline $\mathrm{Aa} 2$ & -446 & -1583 & -809 & -29 & -857 & -43 & -402 & -682 & -20 & -750 & -39 \\
\hline $\mathrm{Ba} 2$ & -396 & -1348 & -663 & -18 & -734 & -38 & -352 & -536 & -8 & -672 & -33 \\
\hline $\mathrm{Ca} 2$ & -337 & -1055 & -500 & -5 & -593 & -31 & -293 & -372 & * & -486 & -33 \\
\hline $\mathrm{Ab} 2$ & -202 & -627 & -368 & -29 & -395 & -46 & -191 & -336 & -25 & -368 & -44 \\
\hline $\mathrm{Bb} 2$ & -182 & -538 & -279 & -9 & -331 & -36 & -171 & -247 & -3 & -305 & -33 \\
\hline $\mathrm{Cb} 2$ & -151 & -390 & -172 & $*$ & -246 & -40 & -140 & -140 & * & -219 & -45 \\
\hline Ac2 & -116 & -290 & -223 & -36 & -238 & -53 & -114 & -215 & -34 & -231 & -53 \\
\hline $\mathrm{Bc2}$ & -110 & -274 & -163 & -4 & -202 & -37 & -108 & -155 & -2 & -195 & -35 \\
\hline $\mathrm{Cc} 2$ & -95 & -199 & -84 & $*$ & -147 & -54 & -92 & -76 & * & -140 & -45 \\
\hline
\end{tabular}

a) $E_{\mathrm{a}}^{*}(011)$ has been calculated on the basis of $d_{022}$.

(b) Specific surface energy (in $\mathrm{mJ} \mathrm{m}^{-2}$ )

\begin{tabular}{|c|c|c|c|c|c|c|c|c|c|c|}
\hline \multirow[t]{4}{*}{ Model } & \multicolumn{10}{|c|}{ Equilibrium form } \\
\hline & \multicolumn{5}{|c|}{ Disordered } & \multicolumn{5}{|c|}{ Ordered (i) } \\
\hline & \multirow{2}{*}{$\begin{array}{l}(011) \\
E_{\mathrm{a}}\end{array}$} & \multicolumn{2}{|l|}{$(010)$} & \multicolumn{2}{|l|}{$(031)$} & \multirow{2}{*}{$\begin{array}{l}011) \\
E_{\text {a }}\end{array}$} & \multicolumn{2}{|l|}{$(010)$} & \multicolumn{2}{|l|}{$(031)$} \\
\hline & & $\overline{E_{\mathrm{a}}}$ & $\overline{\mathrm{HI}}$ & $\overline{E_{\mathrm{a}}}$ & HI & & $\overline{E_{\text {a }}}$ & $\mathrm{HI}$ & $\overline{E_{\mathrm{a}}}$ & HI \\
\hline$\overline{\mathrm{Aa} 2}$ & -1852 & -2364 & $*$ & -2323 & * & -1669 & -1990 & * & -2124 & -5.9 \\
\hline $\mathrm{Ba} 2$ & -1647 & -1938 & $*$ & -1964 & $*$ & -1463 & -1564 & * & -1765 & -7.7 \\
\hline $\mathrm{Ca} 2$ & -1401 & -1461 & * & -1555 & -0.5 & -1217 & -1087 & * & -1357 & -1.0 \\
\hline $\mathrm{Ab} 2$ & -839 & -1071 & * & -1056 & -0.3 & -793 & -977 & * & -1007 & -3.8 \\
\hline $\mathrm{Bb} 2$ & -753 & -810 & * & -856 & -2.4 & -707 & -716 & $*$ & -815 & -6.0 \\
\hline $\mathrm{Cb} 2$ & -627 & -498 & $*$ & -603 & -1.5 & -581 & -404 & $*$ & -554 & -6.9 \\
\hline $\mathrm{Ac} 2$ & -483 & -647 & * & -631 & -1.2 & -471 & -623 & * & -619 & -2.2 \\
\hline $\mathrm{Bc} 2$ & -457 & -468 & $*$ & -506 & -1.6 & -446 & -445 & * & -494 & -2.9 \\
\hline $\mathrm{Cc} 2$ & -391 & -239 & $*$ & -334 & -3.4 & -379 & -215 & * & -322 & -5.3 \\
\hline
\end{tabular}


computed attachment energies, the growth forms have been constructed. In fig. 3a the effect of the charge distribution is presented horizontally for the charge $q_{\mathrm{H}_{0}}$, and vertically for the charge $q_{\mathrm{O}}$. Low effective charges of oxygen and $\mathrm{H}_{0}$ are favourable for the development of a prismatic

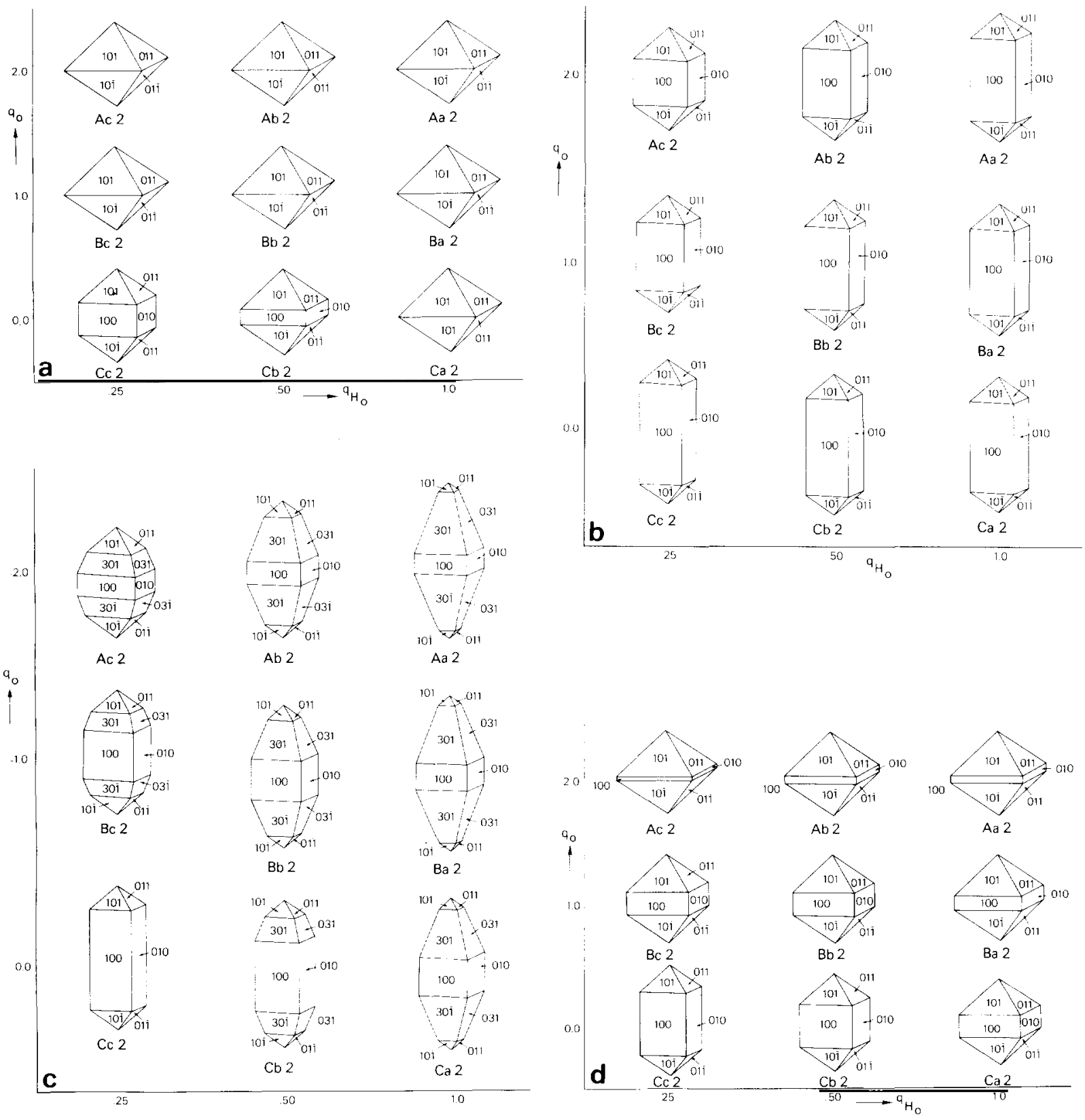

Fig. 3. All presented forms are drawn with the same volume: (a) theoretical growth form of ADP (model **2); (b) theoretical growth form of ADP (model $* * 2$ ) based on the halving of the slices $d_{011}$; (c) theoretical growth form of ADP (model $* * 2$ ) based on the halving of the slice $d_{011}$, and the presence of an $\mathrm{S}$ form $\{031\}$ due to the activities of impurities; (d) theoretical equilibrium form of ADP (model **2) 
habit. Otherwise the bipyramidal habit is dominant, while in most of the models the HI of (010) and (031) is high.

\subsection{Ordered configuration}

The values of the ordered (i) configuration are also listed in table 4a. The effect of the ordering on the growth form is almost negligible. Although the HI of $(010)$ is more negative than in the corresponding disordered models, only the growth form of model $\mathrm{Ca} 2$ changes by the addition of $\{100\}$, just as in the case of the disordered $\mathrm{Cb} 2$ model. In those models with $q_{\mathrm{O}}=0$, where the completely covalent $\mathrm{P}-\mathrm{O}$ bonds do not contribute to the ionic attachment energies, the ordering process of $\mathrm{H}_{0}$ produces significant changes in the attachment energy.

\section{Equilibrium forms}

\subsection{Introduction}

Although the conditions of the crystal growth experiments, in which ADP crystals have been grown, do not reach the equilibrium state, the effect of an equilibrium-like situation is studied by constructing the equilibrium form as a Wulf plot of the specific surface energies. The specific surface energy is given by the following equation

$\gamma_{h k l}=k Z_{\mathrm{p}} d_{h k l}\left(2 V_{\mathrm{p}}\right)^{-1} \sum_{m=1}^{\infty} m E_{m}$,

where $\gamma_{h k l}=$ specific surface energy in $\mathrm{mJ} \mathrm{m}^{-2}$, $k=166.0362$ (conversion factor to obtain $\mathrm{mJ} \mathrm{m}^{-2}$ from $\mathrm{kJ} \mathrm{mol}^{-1}$ ), $Z_{\mathrm{p}}=$ number of molecules in the primitive unit cell, $d_{h k l}=$ interplanar spacing of slice $d_{h k l}, E_{m}=$ partial attachment energy of slice $m$, and $V_{\mathrm{p}}=$ volume of primitive cell.

\subsection{Equilibrium forms}

The values of the specific surface energies of the disordered and ordered $* * 2$ models are listed in table 4 . The equilibrium forms for the disordered $* * 2$ models are shown in fig. $3 \mathrm{~d}$. The prism $\{100\}$ always absent from the growth form is present on the equilibrium form of all the models. The equilibrium forms of the models with low $q_{\mathrm{O}}$ and $q_{\mathrm{H}_{0}}$ have a pronounced prismatic habit.

In two models, i.e. $\mathrm{Aa} 2$ and $\mathrm{Ba} 2$, the $\mathrm{S}$ face (031) is present. The area of that crystal face is so small that it cannot be drawn properly on the crystals presented in fig. $3 \mathrm{~d}$. In all other models the (031) HI has relatively large negative values, in any case not exceeding a few percent.

The ordered equilibrium forms vary only slightly from the disordered ones; only $\{100\}$ is slightly more prominent. The $\{031\}$ is never present on the equilibrium form of the ordered models.

\section{Discussion}

\subsection{The prismatic habit of $A D P$}

\subsubsection{Introduction}

So far the most surprising result of the calculations is the absence of the prism $\{100\}$ from the growth forms of almost all models. This is in sharp contrast with the morphology of crystals as grown in laboratory experiments. The normally prismatic habit of the latter ones is only present on the computed growth forms of the $\mathrm{Cc} 2$ and $\mathrm{Cb} 2$ models. The ordering of the $\mathrm{H}_{0}$ boundary ions does not result in the presence of the prism $\{100\}$ on the growth form of any other models.

On the theoretical equilibrium forms the $\{100\}$ is always present. If becomes more pronounced by the ordering of the hydrogen atoms $\mathrm{H}_{0}$ present on the slice boundaries. However, the prismatic habit of the crystals grown in laboratory experiments can hardly be explained by a state of equilibrium during the growth.

\subsubsection{The presence of the prism $\{100\}$ as a function of $q_{O}$ and $q_{H_{O}}$}

The conditions under which the prism $\{100\}$ is energetically stable depend on the effective charges $q_{\mathrm{O}}$ and $q_{\mathrm{H}_{0}}$. As the energies depend on the valence $q$ according to a parabolic function only three calculated energy values corresponding to differ- 


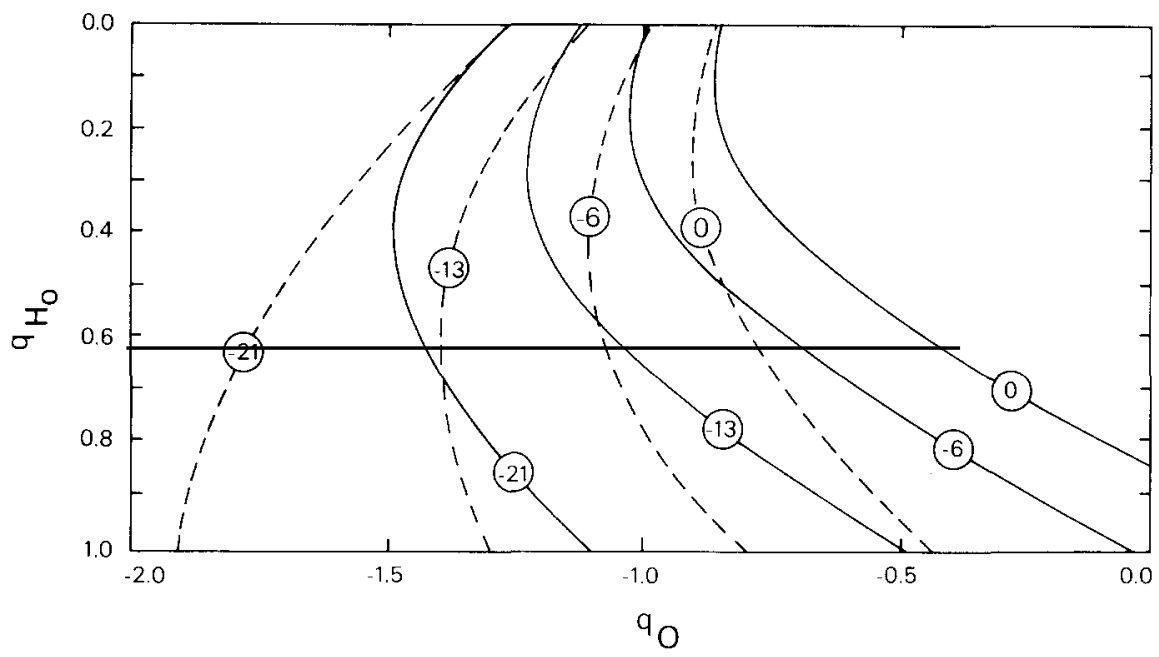

Fig. 4. The presence of the crystal form $\{100\}$ as a function of the charge distribution within the $\left[\mathrm{H}_{2} \mathrm{PO}_{4}\right]^{-}$ion $\left(q_{\mathrm{H}_{0}}\right.$ and $\left.q_{\mathrm{O}}\right)$ is shown for the model Aa2 by lines of equal hypermorphological indices (numbers in circles) as follows: ( $\longrightarrow$ ) disordered model $(-\ldots$,$) ordered model.$

ent $q$ values have to be known in order to define this function. Once this function is known, the energy for any value of $q$ can be calculated. This has been performed for the attachment energies of $\{100\}$ and $\{011\}$ in the models $* * 2$, by varying continuously the charges $q_{\mathrm{O}}$ and $q_{\mathrm{H}_{0}}$. In fig. 4 the results of these computations are presented as curves of equal hypermorphological indices. The solid lines indicate the HI of $\{100\}$ for the disordered models as a function of the charges $q_{\mathrm{O}}$ and $q_{\mathrm{H}_{0}}$, while the dashed ones are drawn for the ordered models. The HI of $0 \%$ coincides with a ratio $E_{\mathrm{a}}^{010} / E_{\mathrm{a}}^{011}=1.41$, which is exactly the limiting value allowing $\{100\}$ to be present.

As these ratios increase, the HI values become more negative making the presence of $\{100\}$ less likely. From fig. 4 one can deduce that the presence of $\{100\}$ is limited to the charge distribution $0.9<q_{\mathrm{O}}<0$ and $0<q_{\mathrm{H}_{0}}<0.8$.

\subsubsection{Halving of the slice $d_{011}$}

Hartman and Heijnen [13] described a new growth mechanism for those crystal faces for which more than one slice configuration can be defined. They do not refer to those differences in slice configuration due to more than one possible choice of boundary ions, such as the $\mathrm{H}_{0}$ hydrogens of (010) in the case of ADP. In some crystal struc- tures one or more different slice configurations of a specific slice $d_{h k l}$ can be defined, which differ in height above the reference level by one or more submultiples of $d_{h k l}$. For ADP such a slice is $d_{011}$ [1], which has been described as an elementary growth layer either bounded by positive $\left[\mathrm{NH}_{4}\right]^{+}$ ions or by negative $\left[\mathrm{H}_{2} \mathrm{PO}_{4}\right]^{-}$ions. The attachment energy of the former is $-450 \mathrm{~kJ} \mathrm{~mol}^{-1}$ $\left(d_{011 \mathrm{~A}}\right)$ and of the latter $\left(d_{011 \mathrm{~B}}\right)$ it is $-1185 \mathrm{~kJ}$ mol $^{-1}$ (model Aa1).

The slice $d_{011}$ can be divided into two slices $d_{022}$, marked $\alpha$ and $\beta$ in fig. 2d [1]. These half slices are still $\mathrm{F}$ faces, being parallel to two PBCs and differ from each other in height by $\frac{1}{2} d_{011}$. Their slice energies must be the same, as they are symmetrically equivalent by the presence of twofold symmetry axes. Although ADP is not a centrosymmetric compound, the modified and extended growth mechanism of Hartman and Heijnen [14] can be applied to (011) of ADP, as the (011) slices are nonpolar and stoichiometric.

The relative area occupied by the two different $\mathrm{F}$ configurations $\mathrm{A}$ and $\mathrm{B}$ can be computed from the following Boltzmann distribution:

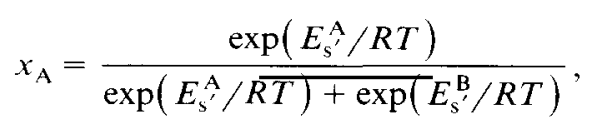


where $x_{\mathrm{A}}=$ relative area of configuration $\mathrm{A}, E_{\mathrm{s}^{\prime}}^{X}$ $=f E_{\mathrm{s}}^{X}$, and $X$ is either $\mathrm{A}$ or $\mathrm{B}$. The correction factor $f$ is equal to the ratio of the experimentally determinated specific surface energy in the solution to the average specific surface energy with reference to vacuum.

The average specific surface energy of ADP in model Aa2 amounts to about $-2200 \mathrm{~mJ} \mathrm{~m}^{-1}$ (mean of the (100), (011) and (031) values), while the experimental value of the surface energy for ADP is given as -6 (average of two values) [15].

Recently Chernov et al. [16], who do not accept that surface diffusion plays a noticeable role during the growth of ADP crystals at the conventionally used supersaturation, obtained experimental surface energies as low as -16 and $-25 \mathrm{~mJ} \mathrm{~m}^{-2}$.

The surface free energy has also been determined from nucleation experiments carried out in solutions with various soluble impurities. The surface energy varies from $-4.304 \mathrm{~mJ} \mathrm{~m}^{-2}$ (pure system) to about $-5.5 \mathrm{~mJ} \mathrm{~m}^{-2}\left(\mathrm{NH}_{4} \mathrm{Cl}\right.$ as impurity) [17].

Computations following eq. (4) on the basis of $-6 \mathrm{~mJ} \mathrm{\textrm {m } ^ { - 2 }}$ lead to a $70 \%$ occupancy of the total slice surface by the slice $d_{011 \mathrm{~A}}$ configuration; based

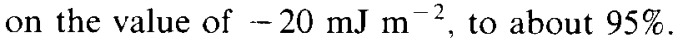

The total area of the slice $d_{011 \mathrm{~B}}$ is, even in the latter situation, large enough to induce the growth of (011) according to halved slices $d_{011}$, i.e. $d_{022}$. The growth rate of (011) will increase and the growth form will change substantially. The growth of (011) will take place at a growth rate directly proportional to $E_{\mathrm{a}}(011)$ at the minimum, and to $E_{\mathrm{a}^{*}}(011)$ at the maximum, which energy is based on the halved slices $d_{022}$ as given in table $4 \mathrm{a}$.

The corresponding growth forms given in fig. $3 \mathrm{~b}$ can be expected under those conditions in which the energetically most favourable slice configuration cannot be realized due to the high crystallization rate. Slowly growing crystals will tend to comply with the conditions of lowest surface energy and should be similar to those forms presented in fig $3 a$.

Dam et al. [18] tried to explain their observations on the tapering of KDP crystals by means of (011) slice energy calculations. They used a simplified point charge model with only $K$ and $P$, neglecting the contribution of hydrogen and oxygen. That contribution can be estimated at about $4 \%$ of the total attachment energy obtained by the present authors. They state incorrectly that the elementary growth layer of (011) cannot be equal to $d_{022}$, because the growth hillocks and etch pits on (011) have a triangular instead of a hexagonal shape. These hexagonally shaped surface figures would, however, form an acute angle between the steps and the crystal surface. Therefore they have a triangular shape being oriented exactly in the same way on both sides. Their energy calculations indicate a positive attachment energy for the $\mathrm{K}^{+}$ions on the (011) slice bounded by $\mathrm{K}^{+}$ions and a negative one for the (011) configuration with phosphate boundary ions, which is not surprising. Their interpretation that the $\mathrm{M}^{+}$ion adsorption will be severely hindered on the $\mathrm{K}^{+}$ion bounded (011) slice surfaces may be correct. Any anion, however, will attach itself on such a surface very easily, after which a cation will be attracted by the local negative charge of the phosphate ion. The attachment energies calculated for individual ions in point charge models equal the attachment energy of that ion on an infinite flat surface. The moment one of the constituent ions attaches itself on this surface, the calculated attachment energy of any other ion belonging to the growth unit no longer reflects the attractive or repulsive energy between that ion and the partly occupied crystal surface. Knowledge of the ion attachment energies can only provide information about which ion has the most favourable interaction energy to attach itself as the first ion on flat surface. The growth of $\{011\}$ can take place by slices bounded either by phosphate or ammonium ions. As has been shown earlier, the phosphate bounded slices are only present on about $5-30 \%$ of the entire $\{011\}$ surface. The relatively low content of $\mathrm{M}^{+}$impurities on $\{011\}$ could be explained by the presence of larger areas bounded by $\mathrm{NH}_{4}^{+}$ions, which is not favourable for $\mathrm{M}^{+}$ adsorption according to Dam et al [18]. Hence it is not possible to draw the conclusion, on the basis of the attachment energy calculations, that the $\mathrm{K}$ bounded slice of (011) is the unique growth layer. On the contrary, the present authors have provided sufficient evidence that the elementary growth layers can be $d_{022}$ thick, for which the 
$\left[\mathrm{NH}_{4}\right]^{+}$bounded slice is energetically more favourable than the phosphate bounded one.

\subsection{The bipyramid $\{031\}$}

Special attention has to be paid to $\{031\}$, of which the specific surface energies are so low that this form is present on the theoretical equilibrium form of disordered models with $q_{0} \leqq-1 \wedge q_{\mathrm{H}_{0}}=$ $1 \wedge q_{\mathrm{H}_{\mathrm{N}}}=0.5$. The form $\{031\}$ is always absent from models ${ }^{* *} 1,{ }^{* *} 3$ and ${ }^{* *} 4$. In the disordered $* * 2$ models the $\{031\}$ specific surface energies and attachment energies are of the same order of magnitude as those of $\{010\}$. Hence the form $\{031\}$ could appear on those growth forms which are based on the $d_{022}$ attachment energies. To that end it would have to change its $S$ character into an $F$ one, because (1) only $F$ forms growing according to a layer mechanism are present on the growth forms and (2) only for $F$ faces is the attachment energy directly proportional to the growth rate. For $\{031\}$ the chain energy is almost equal to the slice energy. Such a situation could give rise to a favourable kink site for the capture of an impurity. An impurity attached to the surface of $\{011\}$ could provide additional strong bonds resulting in an $F$ character of the $\{031\}$. If the existence of $\{031\}$ is theoretically admissible, the growth forms of all the $* * 2$ models shown in fig. $3 \mathrm{c}$ resemble the so-called tapered ADP crystals. All HIs of $\{031\}$ as given in table $3 \mathrm{a}$ are computed for the models based on the attachment energies of the $d_{011}$ slice.

\subsection{Effects of growth units}

The energies have been calculated assuming that all bonds are formed during crystallization, i.e. the crystallization takes place from vapour. As already mentioned earlier, $\left[\mathrm{NH}_{4}\right]^{+}$and $\left[\mathrm{H}_{2} \mathrm{PO}_{4}\right]^{-}$ ions are present in the solution, leading one to suppose that the energies related to the formation of those ions should be subtracted from the computed energy values. This is not necessary because the attachment and specific surface energies are built up of interaction energies of al the constituent ions of the crystallizing unit (ion) with the growing crystal. Hence a correction for the inter-
Table 5

Corrected crystal energies of ADP (model **2)

\begin{tabular}{llrl}
\hline Model & \multicolumn{2}{l}{$E^{\mathrm{corr}}\left(\mathrm{kJ} \mathrm{mol}^{-1}\right)$} & $\begin{array}{l}E_{\mathrm{cr}}^{\mathrm{corr}} \\
\left(\mathrm{kJ} \mathrm{mol}{ }^{-1}\right)\end{array}$ \\
\cline { 2 - 3 } & $\mathrm{NH}_{4}$ & $\mathrm{H}_{2} \mathrm{PO}_{4}$ & \\
\hline $\mathrm{Aa} 2$ & -1833 & -26528 & -1991 \\
$\mathrm{Ba} 2$ & -1833 & -3713 & -1722 \\
$\mathrm{Ca} 2$ & -1833 & -3183 & -1397 \\
$\mathrm{Ab} 2$ & -1833 & -31429 & -1169 \\
$\mathrm{Bb} 2$ & -1833 & -5116 & -1031 \\
$\mathrm{Cb} 2$ & -1833 & -1087 & -843 \\
$\mathrm{Ac2}$ & -1833 & -34257 & -860 \\
$\mathrm{Bc} 2$ & -1833 & -6195 & -793 \\
$\mathrm{Cc} 2$ & -1833 & -418 & -669 \\
\hline
\end{tabular}

nal bonds within the $\left[\mathrm{NH}_{4}\right]^{+}$and $\left[\mathrm{H}_{2} \mathrm{PO}_{4}\right]^{-}$ions is not necessary. However, as far as the crystal or slice energies are concerned, they ought to be corrected for the presence of $\left[\mathrm{NH}_{4}\right]^{+}$and $\left[\mathrm{H}_{2} \mathrm{PO}_{4}\right]^{-}$ions, table 5 , columns 2 and 3 respectively. The values of the crystal energies for all the **2 models are also listed in table 5. These crystal energies calculated from known slice and attachment energies according to eq. (3) are of course function of the charge distribution.

\subsection{Effects of the charge distribution}

The calculation of the growth and equilibrium forms have been calculated for a series of charge distributions. The model $\mathrm{Bb} 2$ is more or less a compromise between the Skorczyk and the Pullman charge distribution models. The completely ionic $\mathrm{Aa}^{*}$ models have always the same habit as the $\mathrm{Bb}^{*}$ ones. The models with the relatively weaker hydrogen bond and the completely covalent $\mathrm{P}-\mathrm{O}$ bond (model $\mathrm{Cc} 2$ ) show mostly a slightly different habit. The effect of the charge distribution on the crystal morphology surprisingly small.

\section{Conclusions}

(1) The crystal forms $\{011\}$ and $\{100\}$, normally present as the only crystal faces on crystals grown in the laboratory, are $\mathrm{F}$ faces. The outer surface structure of $\{100\}$ consists of both positive and negative ions, while the surface of $\{011\}$ is 
occupied by either positive or negative ions. The former configuration is the most stable one based on energy calculations.

(2) Calculations in different point charge models show that $\{100\}$ is present on the growth form if $-0.9<q_{\mathrm{O}}<0$ and $0<q_{\mathrm{H}_{0}}<0.8$. Its morphological importance increases as the absolute values of the electric charges $q_{\mathrm{O}}$ and $q_{\mathrm{H}_{0}}$ decrease, which can be explained in terms of increasing covalency of the $\mathrm{P}-\mathrm{O}$ bond and weakening of the $\mathrm{O}-\mathrm{H}_{0} \ldots \mathrm{O}$ hydrogen bond.

(3) The fact that (011) can grow with elementary growth layers, which are only $\frac{1}{2} d_{011}$ thick, introduces in the growth forms of all the models a clearly pronounced prismatic habit caused by the presence of both $\{100\}$ and $\{011\}$. This could explain the prismatic habit of relatively rapid growing crystals, as grown in laboratory experiments.

(4) Different charge distributions within the $\left[\mathrm{NH}_{4}\right]^{+}$ion do not influence the theoretical growth forms.

(5) The form $\{031\}$ is an $\mathrm{S}$ form, and cannot be present on the theoretical growth form. The presence of $\{031\}$ on crystals grown in the laboratory must be due to external factors.

(6) As $\mathrm{KDP}\left(\mathrm{KH}_{2} \mathrm{PO}_{4}\right)$ is isomorphous with ADP the conclusions about the crystal morphology of ADP are also valid for KDP.

\section{Acknowledgements}

The authors thank Professor P. Hartman (State University of Utrecht) for his stimulating and helpful criticism, which improved the paper to a great extent. One of us (M.A.) is indebted to Professor M. Font-Altaba (University of Barcelona), who supervised her PhD Thesis on ADP, for his helpful discussions. Further she ackowledges the financial support from the Consejo Superior de Investigaciones Cientificas and the Comissió Interdepartamental de Recerca I Innovació
Tecnològica of the Generalitat of Catalunya, which enabled her to stay at the State University of Utrecht during the summers of 1981 and 1982.

This research was also financially supported by CSIC (number 555/85) at the CAICYT PR 840102 project.

The authors are grateful to Dr. C.S. Strom for providing her APL computer programs for the computation of the parabolic dependence of the surface energies as a function of the charges. She also kindly corrected the English manuscript.

\section{References}

[1] M. Aguiló and C.F. Woensdregt, J. Crystal Growth 69 (1984) 527.

[2] P. Hartman, in: Crystal Growth: An Introduction, Ed. P. Hartman (North-Holland, Amsterdam), 1973) ch. 14.

[3] J.W. Mullin, A. Amatavivadhana and M. Chakraborty, J. Appl. Chem. 20 (1970) 153.

[4] P. Hartman and P. Benneman, J. Crystal Growth 49 (1980) 145.

[5] C.F. Woensdregt, Z. Krist. 162 (1983) 239.

[6] C.S. Strom, J. Crystal Growth 46 (1979) 85.

[7] P. Hartman, Acta Cryst. 9 (1956) 569.

[8] C.F. Woensdregt, Energy: A Fortran IV program to compute surface energies in an electrostatic point charge model (Leiden University, 1971).

[9] L.G. Sillén, in: Treatise on Analytical Chemistry, Part 1, Vol. 1, Eds. E.M. Kolthoff, Ph.J. Elving and E.B. Sandell (Interscience Encyclopedia, New York, 1959) section B, ch. 8.

[10] A. Pullman, B. Pullman and H. Berthod, Chim. Acta 47 (1978) 175.

[11] R. Skorczyk, Acta Cryst. A32 (1976) 447.

[12] J. 't Hart, Can. Mineral. 16 (1978) 547.

[13] P. Hartman and W.M.M. Heijnen, J. Crystal Growth 63 (1983) 261.

[14] W.M.M. Heijnen, PhD Thesis, Utrecht University (1986) ch. II.

[15] O. Söhnel, J. Crystal Growth 57 (1982) 101.

[16] A.A. Chernov, L.N. Rashkovich and A.A. Mkrtchan, J. Crystal Growth 74 (1986) 101.

[17] S. Nagalingam, S. Vasudevan and P. Ramasamy, Crystal Res. Technol. 16 (1981) 647.

[18] B. Dam, P. Bennema and W.J.P. van Enckevort, J. Crystal Growth 74 (1986) 118. 